Bioscientia Medicina: Journal of Biomedicine \&

Translational Research

Journal Homepage: www.bioscmed.com

\title{
Coronary Microvascular Dysfunction
}

\section{Doharjo Manullang ${ }^{*}$, Imran Soleh ${ }^{2}$, Rukiah Chodilawati ${ }^{2}$, Syamsu Indra $^{2}$, Ferry Usnizar $^{2}$, Erwin Sukandi², Taufik Indrajaya ${ }^{2}$, Ali Ghanie ${ }^{2}$}

1 Subspesialized Training, Cardiovascular Division, Department Internal Medicine, Faculty of Medicine, Universitas Sriwijaya, Palembang, Indonesia

2 Cardiovascular Division, Department Internal Medicine, Faculty of Medicine, Universitas Sriwijaya, Palembang, Indonesia

\section{A R T I C L E I N F O}

Keywords:

Coronary microvascular dysfunction

Mechanism

Diagnostic test

Treatment

*Corresponding author:

Doharjo Manullang

E-mail address:

\section{doharjomanullang@yahoo.com}

All authors have reviewed and approved the final version of the manuscript.

https://doi.org/10.32539/bsm.v5i12.428

\begin{abstract}
A B S T R A C T
Coronary microvascular dysfunction (DMK) is a condition of patients who are accompanied by complaints of chest pain where the results of coronary angiography examination are normal and this is almost $49 \%$ with $60 \%$ of patients diagnosed with DMK. Another study said that about $40 \%$ of patients with DMK showed coronary flow reserve (CFR <2) of about $40 \%$ and the WISE study (Women's Ischaemia Syndrome Evaluation) showed that about $47 \%$ of patients with chest pain had normal coronary arteries. DMK can be divided into 4 groups; DMK with no coronary arterial disease (CAD) obstruction and myocardial disease, DMK with myocardial disease where this occurs due to remodeling of intramural coronary arteries, DMK with CAD (coronary arterial disease) or acute myocardial infarction with or without ST segment, iatrogenic typhoid DMK occurs after coronary recanalization caused by vasoconstriction and distal embolization. The mechanism of action of DMK can be caused by several factors, namely endothelial dysfunction, smooth muscle dysfunction, decreased diastolic perfusion time, damage to blood vessels and damage to the vascular and microvascular remodeling. And to enforce this DMK, there are several tests carried out in diagnosing the disease, some of which are invasive and non-invasive so that by enforcing the diagnosis of this disease, treatment for DMK can be done immediately and optimally.
\end{abstract}

\section{Introduction}

The relationship between myocardial ischemia and obstructive atherosclerosis of the epicardial coronary arteries has been studied previously and coronary angiography has shown an association of severity with coronary artery disease. In some cases, patients come with complaints of chest pain. However, a coronary angiography examination was found to show normal results. Several other cardiovascular examinations, found coronary microvascular dysfunction (DMK) in this patient or also known as microvascular angina. Microvascular angina has a high prevalence of cardiovascular disease, including a high frequency of hospitalization with concomitant heart failure, sudden death due to heart problems, and myocardial infarction. In the past 2 decades, there have been several previous studies that have reported the examination and assessment of DMK both invasive and non-invasive examinations to explain the occurrence of DMK which includes abnormalities of the function and structure of the coronary microcirculation that occur in several clinical conditions that show as a marker of the risk of myocardial ischemia. and also as a therapeutic target to be given. 1

\section{Epidemiology}

Several studies have shown that patients who 
clinically support coronary angiography have no stenosis and this is almost $49 \%$ with $60 \%$ of patients diagnosed with DMK. A study revealed that patients with diabetes mellitus had a prevalence of 59\% after testing for adenosine and acetylcholine. There is also another study that stated that about $40 \%$ of patients with DMK showed coronary flow reserve/CFR <2 (Table 1) and the WISE study (Women's Ischemia Syndrome Evaluation) showed that $47 \%$ of patients with chest pain had normal coronary arteries (Figure 1). 2-4,10

Table 1. Prevalence of coronary microvascular dysfunction ${ }^{2}$

\begin{tabular}{|c|c|c|c|c|c|c|}
\hline \multicolumn{7}{|c|}{ Prevalence of microvascular dysfunction in observational studies by examination modality } \\
\hline Author & Year & Test & $\begin{array}{l}\text { Sample } \\
\text { size }\end{array}$ & $\begin{array}{l}\text { Prevalence } \\
\text { of CMD (\%) }\end{array}$ & $\begin{array}{c}\text { Male } \\
(\%)\end{array}$ & $\begin{array}{c}\text { Female } \\
(\%)\end{array}$ \\
\hline Hasdai et al & 1988 & Coronary reactive test & 203 & 59 & - & - \\
\hline Reis et al & 2001 & Coronary reactive test & 159 & 47 & - & 47 \\
\hline Sade et al & 2009 & CFR $<2$ & 68 & 40 & - & 40 \\
\hline Sicaria et al & 2009 & $\mathrm{CFR}<2$ & 394 & 22 & 25 & 19 \\
\hline Cassar & 2009 & Coronary reactive test & 367 & 63 & 61 & 65 \\
\hline Wei et al & 2012 & Coronary reactive test & 293 & 49 & - & 49 \\
\hline Murthy et al & 2014 & PET & 1218 & 53 & 51 & 54 \\
\hline Sara et al & 2015 & Coronary reactive test & 1439 & 64 & 60 & 66 \\
\hline Mygind et al & 2016 & $\mathrm{CFR}<2$ & 919 & 26 & - & 26 \\
\hline
\end{tabular}

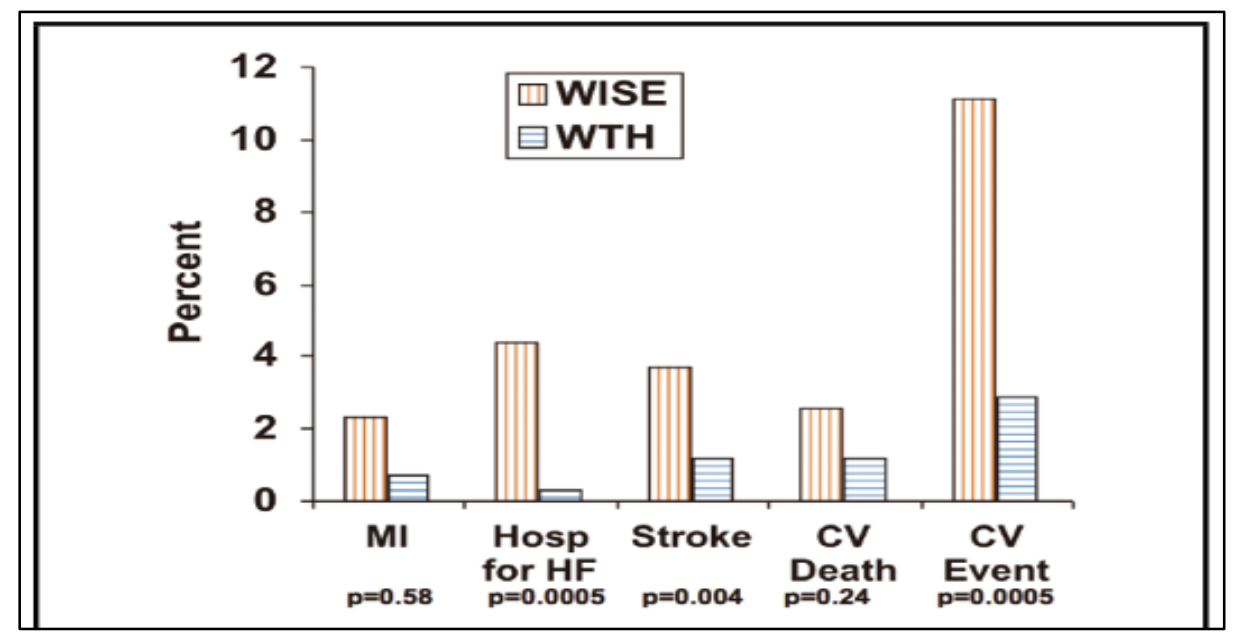

Figure 1. Comparison of average cardiovascular events and mortality between patients Remarks: WISE (Women's ischemia syndrome evaluation) and WTH (women take heart study) patients; CV (cardiovascular); HF (heart failure); MI (myocardial infarction). ${ }^{2}$ 


\section{Coronary microvascular dysfunction without coronary artery disease and myocardial disease Smoking}

Smoking is a risk factor for cardiovascular disease that will affect coronary and peripheral blood flow where endothelial dysfunction in brachial arteries and coronary arteries can occur in heavy smokers. Microvascular dysfunction in asymptomatic smokers with no CAD approach found their CFR decreased by about $21 \%$ compared to non-smokers as controls. But the administration of vitamin $\mathrm{C}$ showed as an antioxidant can restore normal CFR in smokers but the effect is not significant in people who do not smoke (control subjects).4-10

\section{Hyperlipidemia and obesity}

Reduced coronary blood flow reserves in asymptomatic people with hypercholesterolemia where on angiographic examination found normal arteries, this subject can be considered to develop strategies in terms of lowering cholesterol levels. Decreased blood flow reserves have been noted by PET in patients with hyperlipidaemia without symptoms of non-obstructive CAD where a decrease in coronary blood flow correlates with total and LDL cholesterol levels so that treatment of hyperlipidemic patients, with fluvastatin or pioglitazone has been shown to increase blood flow reserves. Obese patients without comorbidities may have lower myocardial blood flow than non-obese. Endothelium damage in obese patients depends on coronary vasomotion in overweight patients with an approximation of myocardial blood flow which can be seen from the vasodilator capacity of dipyridamole. $8,9,10$

\section{Diabetes mellitus}

There is a relationship between diabetes mellitus and coronary risk factors such as obesity, dyslipidemia, and hypertension. The direct effect of vascular changes in diabetes, especially on endothelial function, can increase vasoconstriction and thrombosis. The reserve of coronary blood flow is impaired in diabetic patients, which is an early sign of atherosclerosis. A recent study showed that coronary microvascular dysfunction is characterized in response to adenosine (endothelium vasodilation). $\cdot 9,10$

\section{Microvascular angina}

Patients without obstructive atherosclerotic plaque and myocardial disease accompanied by chest pain are known as cardiac syndrome X (CSX). The hypothesis is that ischemic chest pain in which ST depression is seen during induced chest pain is usually due to stress on myocardial perfusion and reduced coronary endothelial vasodilation associated with myocardial ischemic metabolism but other studies have failed to find an abnormal approach to myocardial blood flow or reserve blood flow. or ischemia or function during ischemic stress. But the point is that patients with syndrome $\mathrm{X}$ show decreased coronary and metabolic flow reserves in myocardial ischemia, coronary myocardial dysfunction that may be caused by angina, which is the basis for the findings of angina in the diagnosis of microvascular angina. Maseri et al., reported that in patients who have focal ischemia in a small portion of the myocardium scattered throughout the myocardium, it is caused by prearteriolar dysfunction. Bottcher et al., stated that the examination of N-Ammonia PET in assessing blood flow in CSX patients was impaired during the stress vasodilator dipyridamole was performed. Patients presenting with chest pain in the absence of significant obstructive CAD demonstrated an association of decreased myocardial perfusion from the epicardium to the endocardium, and one study demonstrated an association between elevated CRP levels and reduced blood flow reserve/CFR (PET) in CSX patients where the inflammatory process is a major factor. important in the pathogenesis of chest pain in CSX patients.

Coronary microvascular dysfunction with chest pain syndrome is more common in women than in men and a reduction in CFR is found which is associated with vasoreactivity and vascular remodeling. However, recent data indicate that diagnosis of coronary microvascular dysfunction is associated with increased morbidity and mortality, especially in women. The WISE (Women's ischemia syndrome evaluation) study stated that women without obstructive CAD found decreased CFR associated with adverse clinical outcomes. ${ }^{11}$

\section{Aging}

The old process occurs in coronary blood flow reduction (CFR) in which patients over 60 years of age 
have an increase in basal flow that causes an increase in cardiac workload generated by the increase in systolic blood pressure.

\section{Microvascular dysfunction with myocardial disease Hypertrophic cardiomyopathy}

Symptoms and signs of myocardial ischemia are common in patients with hypertrophic cardiomyopathy despite normal coronary artery angiography. Myocardial ischemia can lead to severe complications of hypertrophic cardiomyopathy such as ventricular arrhythmias, sudden death, and diastolic dysfunction. 15 years ago a study stated that the reserve blood flow was not only hypertrophied in the septum but also hypertrophied the left ventricular wall. In addition, the severity of coronary microvascular dysfunction in affected patients is an independent long-term predictor of long-term clinical deterioration and cardiovascular death. $8-10$

\section{Dilated cardiomyopathy}

A markedly reduced coronary flow reserve is seen in patients with dilated cardiomyopathy. In patients with dilated cardiomyopathy, coronary microvascular dysfunction is an independent predictor of cardiac events associated with an increased risk of death and further heart failure. 8-10

\section{Secondary cardiomyopathy}

Abnormal coronary flow reserve in essential hypertensive patients with normal angiography of coronary arteries without left ventricular hypertrophy. Coronary microvascular dysfunction in hypertensive patients is not necessarily correlated with the degree of left ventricular hypertrophy. Regional coronary microvascular dysfunction indicates that patients with abnormal patterns of myocardial electrical depolarization or repolarization or regional myocardial ischemia during these conditions need high blood flow. 8-10

\section{Aortic stenosis}

The development of left ventricular hypertrophy in patients with aortic stenosis (SA) is an adjustment response in that it reduces the pressure on the left ventricular wall. Some of the associated changes in the hypertrophic process also affect the coronary circulation and patients with AS have a reduced coronary flow reserve despite normal angiographic examination of the coronary arteries. Unfavorable hemodynamic changes such as high left ventricular pressure, low coronary perfusion pressure compared to intracavitary pressure and increased extravascular pressure result in reduced coronary flow reserve and minimal increase in coronary resistance. Pathologically, microvascular dysfunction occurs period myocyte fibrosis and a decrease in the number of vessel resistance per unit. A study revealed the severity of coronary microvascular dysfunction related to aortic valve area, hemodynamic load, diastolic perfusion and left ventricular mass. Reduced extravascular compression and increased diastolic perfusion time are the main mechanisms in increasing myocardial blood flow and coronary flow reserve after aortic valve replacement. $8-10$

\section{Microvascular dysfunction with coronary artery obstruction}

\section{Stable coronary artery disease}

Studies in single CAD patients with normal left ventricular function have noted coronary flow reserve with normal coronary artery angiography. In patients with stable angina, distal coronary microvascular dysfunction up to coronary stenosis has an important role in inhibiting ischemia. In the absence of epicardial coronary artery stenosis this may be explained by coronary microvascular dysfunction including collateral circulation. The distal portion of coronary microvascular dysfunction to the development of severe coronary stenosis is usually caused by 2 mechanisms, namely inappropriate subepicardial pre arteriolar dilatation due to increased myocardial oxygen consumption and pre arteriolar and arteriolar constriction. The role of pre arteriolar and arteriolar constriction determines the ischemic threshold that occurs. ${ }^{8-10}$

\section{Acute coronary syndrome without ST-segment elevation}

In patients with an acute coronary syndrome 
without segment elevation, distal coronary microvascular dysfunction to severe stenosis plays an important role in reducing the severity of myocardial ischemia not only through mechanisms of stable CAD but also through mechanisms in unstable patients. Marzilli et al., stated that in patients with unstable angina where the pain that occurs at rest is associated with a rapid increase in coronary microvascular resistance which can be prevented by the administration of antiplatelet drugs. In addition, the degree of coronary microvascular dysfunction is proportional to the level of systemic C-reactive protein which is a marker of inflammation which indicates that the disorder caused by inflammation is not dependent on risk factors alone.

\section{Acute myocardial infarction with ST-segment elevation}

In patients with acute myocardial infarction, the reduction in coronary blood flow is sufficiently large to impair regional wall motion of the myocardium during contraction. In patients with ST-segment elevation acute coronary syndromes, coronary microvascular dysfunction is responsible for the inability of the previous ischemic area to reperfusion (reflow phenomenon was not observed). This phenomenon is characterized by a lack of microvascular integrity and patency despite the successful opening of the infarcted artery. It arises from pathological changes during ischemia and is exacerbated during reperfusion. Galiuto stated that this phenomenon is grouped into 2 forms, namely structural and functional forms. In the phenomenon in the structural form, there is no flow and components of the blood vessel wall, it looks like a necrotic myocardium with irreversible damage, while the functional form of small blood vessels is still good but the movement of the small blood vessels is lost in the endothelium. Clinically the phenomenon of noreflow has been demonstrated by several studies linking microvascular damage to cardiac remodeling. 10

\section{Iatrogenic coronary microvascular dysfunction Coronary}

vasoconstriction was achieved after coronary angiography where there was a report stating that the coronary flow reserve in the dilated arteries was still suppressed 1 week after the successful coronary angioplasty. Gregorini et al., attributed the epicardial coronary vasoconstriction that has been reported after coronary angioplasty to activation of alpha-adrenergic receptor antagonists administered before the procedure. These alpha-adrenergic receptors stimulate the constriction of small coronary blood vessels. In addition to vasoconstriction, embolization of the coronary microcirculation contributes to coronary microvascular dysfunction in patients undergoing percutaneous intervention and bypass surgery (CABG). Plaque rupture can occur after percutaneous coronary intervention where small plaque materials migrate to other sites and cause small infarcts.

\section{Assessment of coronary microcirculation}

There are currently several measures that rely on quantification of blood flow through the coronary circulation which are commonly used to describe coronary microvascular function. Coronary blood flow is a measurement of the amount of flow through the coronary arteries in $\mathrm{mm} /$ minute. Below are described several techniques for examining coronary blood flow.

\section{Non-invasive examination}

Radiotracer imaging with positron emission tomography (PET), which is an examination that is often performed and has been recognized as being able to assess coronary blood flow at rest and during the vasomotor stress phase, where the radiotracer used is ${ }^{82}$ rubidium, ${ }^{13} \mathrm{~N}-$ ammonia, or $15 \mathrm{O}$-water. However, this examination has limitations because this examination is not all available at the hospital and the examination fee is quite expensive.

Cardiac magnetic resonance imaging (CMR), this tool uses a Gadolinium base which is an indicator of the media to see myocardial perfusion. A recent study of 22 patients without obstructive epicardial artery disease found microvascular dysfunction.

Computed tomography (CT), to see flow quantification obtained with dynamic perfusion images on $\mathrm{CT}$ which uses radio-opaque iodinated contrast as an indicator and has the same ability as CMR in detecting coronary blood flow disturbances. 
Myocardial contrast echocardiography is an examination using a microbial containing gas with a high molecular weight (BM) to produce scattering of the gas. This examination can be used to measure coronary flow velocity, usually from the mid to distal Left Anterior Descending (LAD). It is hoped that these checks will be available with a high level of security and low cost.

Electrocardiogram (ECG), found an initial change in the ST segment on the ECG after 1 hour of reperfusion therapy in cases of acute myocardial infarction. There are several methods proposed where if the ST segment resolution is $<50 \%$ or $>70 \%$ from the baseline, this can be an indicator of no-reflow and the prognosis is poor.

\section{Invasive examination}

Angiography, which in the case of microvascular obstruction from acute myocardial infarction (AMI) can be assessed during angiography using TIMI (thrombolysis in myocardial infarction flow rates) which are divided into TIMI Flow 0 means there is no antegrade flow in the occluded artery; TIMI flow 1 means slow flow in an occluded artery and incomplete filling of the flow to the distal artery; TIMI flow 2 means that there is slow partial reperfusion only distal flow filling is adequate; TIMI flow 3 means that the flow perfusion is normal and distal filling is sufficient.

All the above examination techniques can not assess the quantity of blood flow but visually assess myocardial flow and visual assessment based on contrast flow. The TIMI flow grade used in assessing epicardial coronary flow after reperfusion was TIMI flow 0-2 associated with microvascular obstruction. The use of contrast media can further evaluate the level of myocardial blush visually in the microcirculation, which is a well-known system category for viewing the intensity of flow in myocardial tissue after contrast injection. This system can also assess obstruction of the microvasculature. ${ }^{12-16}$

Coronary reactivity testing, this examination can be assessed during angiography by using acetylcholine infusion as a trigger where the endothelium will release nitric oxide in response to acetylcholine so that vasodilation occurs in the endothelium. In endothelium dysfunction, this will be impaired where the opposite occurs, namely the failure of endothelial function of coronary vasomotion which is an important factor as a cause of microvascular dysfunction. The vasomotor response of the epicardial arteries whose diameter can be assessed by quantitative angiography or intravascular ultrasound (IVUS) is the vasomotor response of the microvasculature which can be assessed by coronary flow reserve (CFR). The risk of this test in endothelial dysfunction is the risk of severe vasospasm with acetylcholine use and this is a limitation of coronary reactivity testing. 12-16

Coronary flow reserve is currently the gold standard examination in assessing microvascular dysfunction by using a hyperemic ratio to coronary base flow. In general, a CFR cut-off $<2$ indicates abnormal myocardial perfusion. Conventionally, the CFR is determined using the moderate Doppler technique, with high-frequency intracoronary Doppler used to record the velocity of blood flow where the wire is placed in the proximal segment of the coronary artery and the vessel portion is measured by angiography or by intravascular ultrasound (IVUS). In addition, the CFR can also be determined using a thermodilution intracoronary technique where the wire is equipped with a pressure and temperature microsensor. ${ }^{12-16}$

Fractional flow reserve (FFR), is the ratio of the maximum blood flow in a stenotic artery divided by the maximum blood flow in a healthy artery (2-flow ratio). Because flow is proportional to pressure, pressure can be used as a substitute for maximal hyper meal squamous flow. The pressure in a normal coronary artery is equal to aortic pressure $(\mathrm{Pa})$, so that the FFA value is the same as $\mathrm{PdPa}$ where $\mathrm{Pd}$ is the coronary pressure distal to the stenosis where both can be measured at maximum coronary hyperemia. If the FFR value is 1 , it is said to be normal, but if the FFR value is $0.75-0.80$, it is still said to be a gray area where revascularization does not need to be done immediately, enough with optimal pharmacological treatment.

Coronary blood flow is used in measuring epicardial coronary artery flow but indirectly provides information about flow through the complex microvasculature and this TIMI rating is assigned a score of 0-3 where higher scores indicate better perfusion. Coronary flow reserve is the increase in blood flow achieved from basal coronary perfusion to maximal vasodilation where flow 
resistance is determined by the microvasculature. CFR is determined by measuring maximum hyperemic blood flow divided by coronary or myocardial blood flow at rest (basal flow achieved by intracoronary (intravenous) adenosine infusion and intravenous dipyridamole. The coronary flow reserve is then expressed as the ratio of blood flow during hyperemia to blood flow when resting. In coronary arterial disease (CAD) patients the magnitude of the reduction in coronary flow reserve is directly related to the severity of the stenosis whereas in normal individuals with normal arterial angiography this is a marker of microvascular dysfunction. The normal value of CFR is highly dependent on the technique used and when a $\mathrm{CFR}<2.0$ indicates ischemia. Baseflow conditions have a significant impact on CFR. An initial increase in myocardial blood flow can lead to a lower CFR. ${ }^{12-16}$

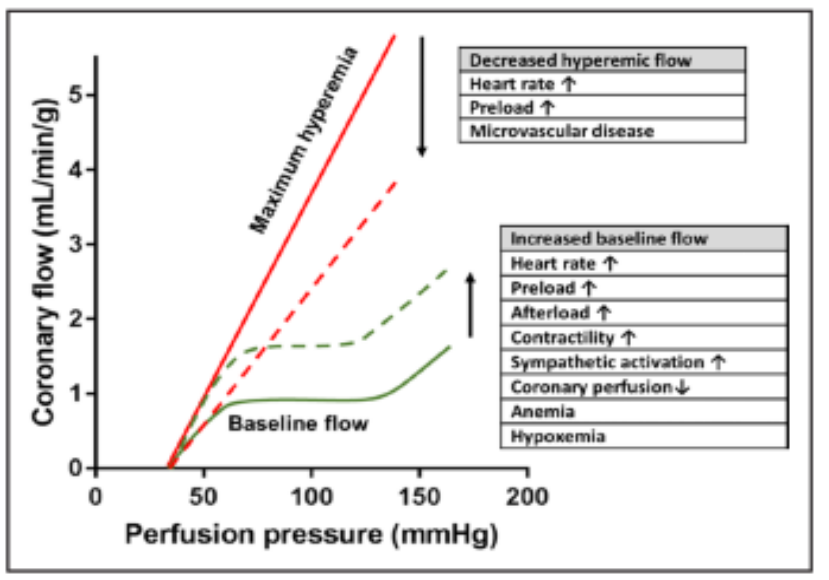

Figure 2. Coronary regulation and determination of coronary flow reserve (CFR).

Remarks: CFR is determined by blood flow maximum hyperemia (red line) divided by basal flow (green line). The increased metabolic demand and reduced oxygen/food supply compensate for the increase in basal coronary flow (dotted green line). The increase in base flow may be due to decreased CFR or reduced hyperaemic flow. Reduced hyperemic coronary flow (dashed red line) is due to reduced diastolic filling time or the presence of coronary microvascular disease. 10
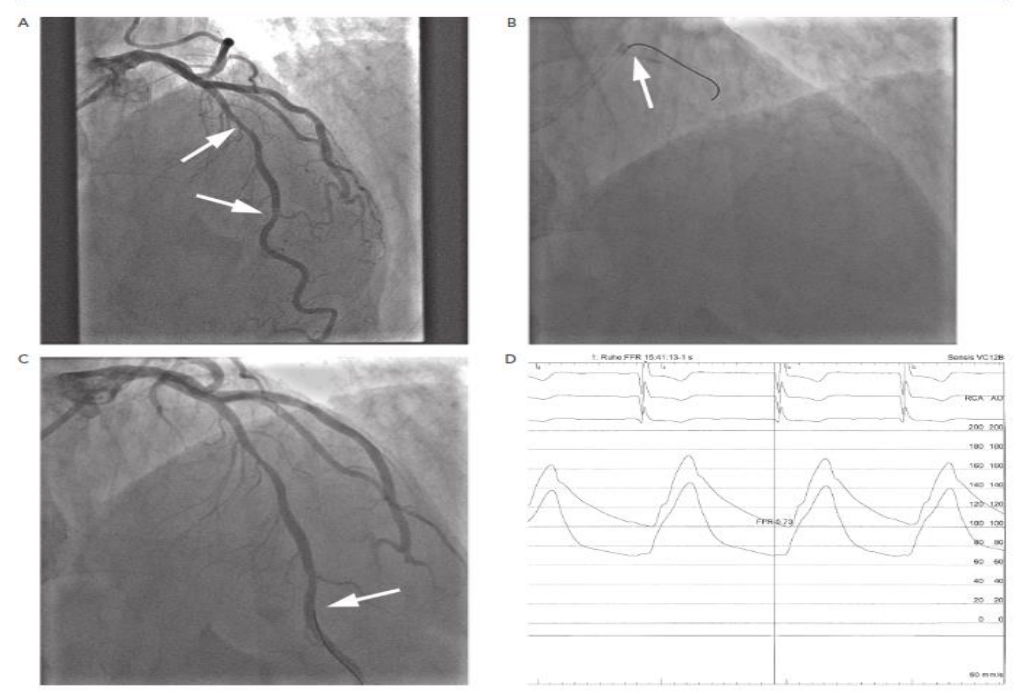

Figure 3. A. angiography showed 2 degrees being on artery stenosis Left Anterior Descending (LAD). B. The position of the FFR wire for measuring pressure is the same where the pressure sensor (arrow line) is placed directly behind the guiding catheter which is used to measure the aortic pressure. C. Position the FFR wire with the pressure sensor (arrow line) directly towards the second distal. D. Intracoronary FFR measurement was seen with a bolus of 80 g adenosine. FFR measurement was 0.73.16 


\section{Management of microvascular dysfunction}

Non-pharmacological management is in the form of lifestyle modification, namely modification of risk factors associated with microvascular dysfunction such as hypertension, obesity, smoking and dyslipidemia. Pharmacological management in the form of drugs that reduce heart rate, namely beta-blockers and ivabradine. Beta-blockers can reduce heart rate, heart contractions and blood pressure, including reducing the oxygen demand of the myocardium. Ivabradine may reduce the rate of SA (sinoatrial) origin. Ivabradine is used when there are contraindications to beta-blockers. Skaldic et al. stated that an increase in hyperemic coronary flow velocity and coronary flow reserve in CAD patients is an indication of an improvement in microvascular function. Villano et al., stated that Ivabradine can improve quality of life when given with anti-ischemic therapy in patients with microvascular angina.

In addition, drugs to reduce coronary microvascular pressure are also used in the treatment, including calcium channel blockers, nitrates and nicorandil. Calcium channel blockers act on the pre arterioles. Verapamil and diltiazem are a class of drugs that can reduce heart rate and can be used in patients who are intolerant of beta-blockers. Meanwhile, the nitrate group is very effective in patients with angina complaints. This drug is associated with reduced levels of nitric oxide in the coronary microcirculation except in the preatriole region.

Nicorandil is a class of drugs that open ATP-sensitive potassium channels, resulting in peripheral dilation. Nicorandil treatment showed a significant reduction in the incidence of no-reflow after reperfusion in patients with acute myocardial infarction and the incidence of slow flow phenomena in percutaneous coronary intervention (IKP). Found slow flow and no-reflow on angiography examination is a sign of microvascular dysfunction. The sulfonylurea effect of nicorandil is to inhibit ATP-K.

Drugs with a positive effect on vascular remodeling used in the treatment of microvascular dysfunction are angiotensin-converting enzyme inhibitors (ACEinhibitors), angiotensin receptor blockers (ARBs), and mineralocorticoid receptor blockers. Long-term therapy with ACE inhibitors shows structural improvement of the coronary arteries which can be seen through endomyocardial biopsy. Angiotensin receptor blockers (ARBs) have a protective effect on vascular remodeling, especially in stroke patients. Mineralocorticoid receptor blockers (MRBs) are a class of drugs that can reduce arterial resistance by decreasing collagen. According to Bavry et al., the use of MRB did not improve coronary endothelial microvascular function in non-obstructive CAD patients. However, another study revealed that giving spironolactone $25 \mathrm{mg}$ and $20 \mathrm{mg}$ / day of enalapril improved coronary microvascular function in patients with type 2 diabetes.

Furthermore, the treatment of microvascular dysfunction can use drugs to increase blood flow, namely cilostazol, pentoxifylline, anticoagulant and antiplatelet. Cilostazol is a selective phosphodiesterase3 (PDE-3) class that inhibits by destroying intracellular cAMP (cyclic adenosine monophosphate). Pentoxifylline is a class of drugs that can reduce viscosity and increase the flexibility of erythrocytes to improve the patient's microcirculation. Anticoagulants are a class of drugs that can increase flow through small, narrow blood vessels. Antiplatelet, in this group Clopidogrel has a strong effect through being an anti-oxidant and antiinflammatory so that it can improve microvascular endothelial function in patients with CAD. This effect has advantages in ACS patients. In addition, drugs that act on myocytes can also be given, namely ranolazine, this class of drugs provides anti-angina effects without affecting heart rate and blood pressure by slowing sodium into the heart myocytes and preventing calcium from being excessive. 17,18

\section{Conclusion}

Coronary microvascular dysfunction in the absence of obstructive CAD is a risk factor for coronary events where this type is reversible and the assessment in this type aims to reduce the burden of risk factors. The complex assessment of microvascular dysfunction increases with the type of obstructive CAD. Lifestyle modification and management of risk factors and comorbidities are of the utmost importance. The use of drugs can be selected depending on the clinical appearance and the mechanism of the disease. 


\section{References}

1. G. Camici $P$ et al. Coronary microvascular dysfunction. N Engl J Med 2007; 356: 830-40.

2. Chen Cheng et al. Coronary microvascular dysfunction: epidemiology, pathogenesis, prognosis, diagnosis, risk factor and therapy. Circ J 2017; 81: 3-11

3. S.Van Thiel Bibi et al. Structure an cell biology of the vessel wall. Krams R, Back Magnus (editors). The ESC Textbook of Vascular Biology. The Oxford University Press. New York. 2017.

4. R. Taqueti V et al. Coronary microvascular disease pathogenic mechanism and therapeutic options. JACC. 2018 November 27; 72(21): 2625-41.

5. Crea Filipo et al. Coronary microvascular dysfunction: an update. European Heart Journal 2014; 35: 1101-1111.

6. G Camici P et al. Coronary microvascular dysfunction: Mechanism and functional assessment. Nat.Rev. Cardiol. (2015); 12:48-62

7. Chabra N. Endothelial dysfunction: a predictor of atherosclerosis, Int $\mathrm{J}$ Med Update, 2009; 4 (1): 33-41.

8. Park KH, Park WJ. Endothelial dysfunction: clinical implications in cardiovascular disease and therapeutic approaches, J Korean Med Sci 2015; 30: 1213-25

9. Picchi A et al. Coronary microvascular dysfunction in diabetes mellitus: A review. World J Cardiol 2010 November 26; 2(11): 377390.

10. Feher Attila, MD, Sinusas A.J. Quantitative assesment of coronary microvascular function. dynamic single-photon emission computed tomography, positron emission tomography, ultrasound, computed tomography, and magnetic resonance imaging. Circ Cardiovasc Imaging. 2017; 10: e0006427.

11. Titterington $\mathrm{J}$ et al. Microvascular angina : an update on diagnosis and treatment. Future Cardiol. 2015; 229-242

12. Xu J, MBBS, et al. Assessing coronary microvascular dysfunction in ischaemic heart disease : little things can make a big difference. Heart Lung and Circulation. 2020; 29, 118127.

13. M. Shah Samit et al. Coronary physiology in the cardiac catheterization laboratory. J.Clin.Med.2019; 8: 225.

14. Lance Gould $\mathrm{K}$ et al. Anatomic versus physiologic assessment of coronary artery disease. JACC. 2013 October 29; 62(18)

15. Ullah M. Fractional flow reserve-A review. Cardiovasc.J.2013; 5(2): 190-200.

16. Achenbach $\mathrm{S}$ et al. Performing and interpreting fractional flow reserve measurement in clinical practing: An expert consensus document. interventional cardiology review. 2017; 12(2): 97-109.

17. Mittal SR et al. Management option for coronary microvascular dysfunction: Present status and future perspectives. Journal of Clinical and Preventive Cardiology 2016; 5: 18-24.

18. Al Rifai Abdullah et al. Microvascular coronary disease: review article. US Cardiology Review 2018;12(1):41-5. Ram Mittal Sita, MD. Review article management option for coronary microvascular dysfunction. Present status and future perspectives. J. Clin Prev Cardiol 2016; 5: $18-24$. 